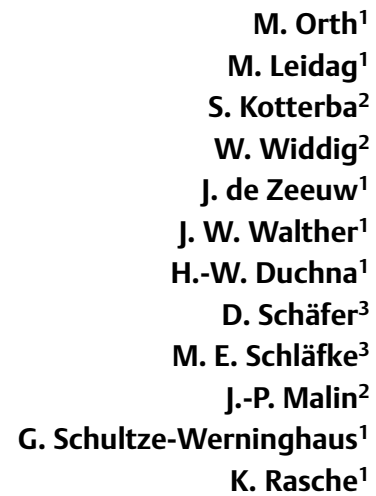

\section{Abschätzung des Unfallrisikos bei obstruktivem Schlafapnoe-Syndrom (OSAS) durch Fahrsimulation}

\author{
Estimation of Accident Risk in Obstructive Sleep Apnea Syndrome (OSAS) \\ by Driving Simulation
}

\section{Zusammenfassung}

Bei Patienten mit obstruktivem Schlafapnoe-Syndrom (OSAS) ist das Unfallrisiko um das 2-7fache erhöht. Untersuchungen zur Unfallhäufigkeit basieren auf anamnestischen Angaben, Unfallstatistiken von Versicherungen und Fahrsimulatoruntersuchungen. Die vorliegende Studie untersucht, ob der Fahrsimulator C.A.R. das erhöhte Unfallrisiko bei Patienten mit OSAS vor und unter Therapie im Vergleich zu Gesunden abschätzen kann. Bei 31 Patienten mit polysomnographisch gesichertem OSAS (Apnoe-Hypopnoe-Index $24,8 \pm 21,5 / \mathrm{h}$ ) wurde eine Fahrsimulatoruntersuchung vor, 2 und 42 Tage nach Einleitung einer CPAPTherapie durchgeführt. Es erfolgte ein Vergleich mit der Fahrsimulatorleistung von 10 gesunden Kontrollen, bei denen ein OSAS mittels Polysomnographie ausgeschlossen wurde. Die Fahrsimulatorleistung unterschied sich insbesondere im Hinblick auf die Häufigkeit von Unfällen (OSAS: 2,7 $\pm 2,0$, Kontrollen: 1,3 $\pm 1,5$, $\mathrm{p}<0,05$ ) und Konzentrationsfehlern (OSAS: 12,4 $\pm 5,1$, Kontrollen: 7,1 $\pm 3,2, \mathrm{p}<0,01$ ) signifikant zwischen OSAS-Patienten und Kontrollen. Unter CPAP konnte die Häufigkeit von Unfällen (OSAS vor Therapie: $12,4 \pm 5,1,2$ Tage CPAP: $1,5 \pm 1,4, \mathrm{p}<0,01 ; 42$ Tage CPAP: $0,9 \pm 1,3, \mathrm{p}<0,001$ ) und Konzentrationsfehlern (OSAS vor Therapie: $12,4 \pm 5,1,2$ Tage CPAP: $6,5 \pm 3,9$, $\mathrm{p}<0,001$; 42 Tage CPAP: $4,9 \pm 3,3, \mathrm{p}<0,001$ ) in der simulierten Fahrsituation sowohl im kurz- als auch mittelfristigen Verlauf signifikant abgesenkt werden. Der Fahrsimulator C.A.R. ist ein adäquates Messinstrument zur Einschätzung des Unfallrisikos bei OSAS und der Effizienz der CPAP-Therapie.

\section{Abstract}

Patients with obstructive sleep apnea syndrome (OSAS) have an accident rate between two and seven times higher than normals. Investigations on accident frequency are based on case history, insurancy reports, and driving simulator investigations. The present controlled study was planned to test whether an increased accident risk could be demonstrated in patients with OSAS before and on CPAP (continuous positive airway pressure)-therapy using the driving simulator C.A.R. Driving simulator performance was investigated in 31 patients with polysomnographically confirmed OSAS (apnea-hypopnea-index $24.8 \pm 21.5 / \mathrm{h}$ ) before, 2 and 42 days after initiation of CPAP and was compared to 10 healthy controls in whom OSAS was excluded by polysomnography. Driving simulator performance was significantly worse in OSAS as compared to normals especially in terms of accident frequency (OSAS: $2.7 \pm 2.0$, controls: $1.3 \pm 1.5, \mathrm{p}<0.05$ ) and concentration faults (OSAS: $12.4 \pm 5.1$, controls: $7.1 \pm 3.2$, p < 0.01). On CPAP accident frequency (OSAS before therapy: $12.4 \pm 5.1,2$ days CPAP: $1.5 \pm 1.4, \mathrm{p}<0.01 ; 42$ days CPAP: $0.9 \pm 1.3, \mathrm{p}<0.001$ ) and frequency of concentration faults (OSAS before therapy: $12.4 \pm 5.1,2$ days CPAP: $6.5 \pm 3.9, \mathrm{p}<0.001$; 42 days CPAP: $4.9 \pm 3.3, \mathrm{p}<0.001$ ) could be lowered significantly both in the short and medium term of therapy. The driving simulator C.A.R. is an adequate tool for the evaluation of an increased accident risk in OSAS-patients and demonstrates the efficiency of CPAP-therapy. und Schlafmedizin (Leiter: Prof. Dr. G. Schultze-Werninghaus),

${ }^{2}$ Neurologische Klinik und Poliklinik (Direktor: Prof. Dr. J.-P. Malin), Bochum,

${ }^{3}$ Abteilung für angewandte Physiologie (Leiterin: Prof. Dr. M. E. Schläfke), Ruhr-Universität Bochum 
Schlafstörungen, die die Tagesbefindlichkeit, insbesondere die Wachheit am Tage beeinflussen, sind für eine Vielzahl von müdigkeitsbezogenen Verkehrs- und Arbeitsunfällen verantwortlich. Die Analyse der Struktur der Unfälle mit Getöteten auf Autobahnen in Bayern im Jahr 1991 zeigt, das bei 49 von 204 Unfällen (entsprechend 24\%) Einschlafen am Steuer das den Unfall auslösende Ereignis darstellte [1]. In den USA wurden die Gesamtkosten für müdigkeitsbedingte Arbeits- und Verkehrsunfälle im Jahr 1988 zwischen 43 und 56 Milliarden Dollar geschätzt [2]. In den USA geht man davon aus, dass das obstruktive Schlafapnoe-Syndrom (OSAS) eine der häufigsten Ursachen gesteigerter Tagesschläfrigkeit darstellt $[3,4]$. Hierbei ist das Vollbild des OSAS jedoch nur der Endpunkt einer Entwicklung, die ihren Anfang beim obstruktiven Schnarchen bzw. Upper Airway Resistance Syndrom hat. Bereits bei diesen Vorstufen des OSAS kann ausgeprägte Tagesschläfrigkeit bestehen. Schon beim habituellen, d.h. schweren Schnarchen ist das relative Risiko, mindestens einen Unfall innerhalb von 5 Jahren zu verursachen auf 3,4 erhöht [5]. Beim OSAS ist das Unfallrisiko mindestens um den Faktor 2-3, in einigen Untersuchungen sogar 7 fach höher als bei Gesunden [5-13].

Untersuchungen zur Unfallhäufigkeit bei OSAS basieren auf anamnestischen Angaben, Unfallstatistiken und Fahrsimulatoruntersuchungen. Hierbei bergen anamnestische Angaben und Unfallstatistiken das Risiko der Unterschätzung der tatsächlichen Unfallhäufigkeit. So verschweigen Patienten mit OSAS im Rahmen des ersten Arztkontaktes $2 / 3$ der tatsächlich verursachten Unfälle $[5,8,14,15]$. Hinzu kommt die Unsicherheit der Unfallstatistik, in die nur die gemeldeten Unfälle eingehen [5]. Fahrsimulatoren werden zunehmend zur Evaluierung der Unfallhäufigkeit bei OSAS angewandt. Mit den bislang eingesetzten Fahrsimulatoren (z.B. Divided Attention Driving Test, Steer clear, STISIM $^{\circledR}$, Ambrocker Vigilanztest, Carsim) können unterschiedliche beim Steuern eines Fahrzeuges erforderliche Aufmerksamkeitskomponenten getestet werden $[16,17]$. Hierzu gehören z. B. die geteilte Aufmerksamkeit (z.B. reger Stadtverkehr) bzw. die Daueraufmerksamkeit, d.h. die längerfristige Aufmerksamkeitsleistung bei hoher Reizfrequenz [18,19]. Mit Hilfe dieser Instrumente gelingt zum einen der Nachweis einer bei OSAS im Vergleich zu Gesunden erhöhten Fehlerrate in der simulierten Fahrsituation sowie eine signifikante Absenkung unter adäquater Therapie $[7,21-28,30]$. Die dargestellten Fahrsimulatoren testen jedoch zumeist nicht den Aufmerksamkeitsaspekt der Vigilanz, d.h. der Aufrechterhaltung der Aufmerksamkeit bei monotonen Umgebungsbedingungen $[18,19]$. Die Einschlafneigung am Steuer imponiert jedoch vor allem bei langen, monotonen Autofahrten, die hohe Anforderungen an die Vigilanz stellen.

Die vorliegende Untersuchung diente der Klärung der Frage, ob der von uns eingesetzte Fahrsimulator C.A.R. geeignet ist, das Unfallrisiko bei OSAS im Vergleich zu Gesunden sowie den Therapieerfolg unter CPAP (continuous positive airway pressure) zu objektivieren.
Bei 31 Patienten mit polysomnographisch (Alice IV, Healthdyne) gesichertem OSAS (29 Männer, 2 Frauen, Alter 55,3 \pm 10,2 Jahre, BMI 29,8 $\pm 6,2 \mathrm{~kg} / \mathrm{m}^{2}$, AHI 24,8 $\pm 21,5 / \mathrm{h}$ ) erfolgte eine Fahrsimulatoruntersuchung vor, 2 Tage und 42 Tage nach Einleitung einer CPAP-Therapie. Der Kontrollzeitpunkt nach 6 Wochen wurde anhand der Empfehlungen zur Begutachtung von Schlaf-Wach-Störungen und Tagesschläfrigkeit ausgewählt [29]. Als Kontrollkollektiv dienten 10 gesunde Probanden ( 8 Männer, 2 Frauen, Alter $55,1 \pm 7,8$ Jahre, BMI $23,1 \pm 3,2 \mathrm{~kg} / \mathrm{m}^{2}$ ), bei denen ein OSAS mittels 2 in der häuslichen Umgebung durchgeführter Polysomnographien (Portable Sleep Monitoring-System ${ }^{\circledR}$, Compumedics) ausgeschlossen wurde.

Der angewandte Fahrsimulator C.A.R. (Computer Aided Risksimulator, Dr. Ing. Reiner Foerst, Gummersbach, Preis ca. DM 100000,-) ist in Abb. 1 dargestellt. Die Fahrkabine ist exakt dem Fahrerplatz eines Pkw nachgebildet. Lenkrad, Blinker und Scheibenwischer, Pedale und Amaturen funktionieren exakt wie in einem Auto. Straße und Verkehrsgeschehen werden über einen Monitor eingespielt, der vor dem Fahrer angebracht ist. Die Fahrgeräusche (Motor, Reifen) werden synchron zum Bildablauf eingespielt. Bremsverzögerung, Beschleunigung und Fliehkraft in den Kurven werden durch die entsprechenden Bewegungen des Fahrersitzes dargestellt. Die technischen Daten des Gerätes sind wie folgt: Länge 2,50 m, Breite 0,80 m, Höhe 1,60 m, Gewicht $500 \mathrm{~kg}$. Mit Hilfe des Computerprogramms können unterschiedliche Fahrbedingungen wie z.B. Alkohol- oder Schlechtwetterprogramm simuliert werden. Neben der computergestützten Auswertung (Anzahl der Unfälle, Reaktionszeit, Gesamtfahrzeit, gefahrene Distanz) erfolgte eine personelle Überwachung während der einstündigen Fahrt sowie eine exakte Analyse der Konzentrationsfehler (z.B. Fehlbedienung von Scheibenwischer oder Lichtanlage) und der Abweichungen von der exakten Fahrspur. Zur Gewöhnung an die simulierte Fahrsituation erfolgte zunächst eine Einweisung der Patienten und der Kontrollpersonen durch den Untersucher. Anschließend wurde zur Adaptation an das System eine 15-minütige Fahrsituation simuliert, erst danach erfolgte die eigentliche Messung. Die der vorliegenden Untersuchung zugrunde liegenden Fahrbedingungen sind in Tab. 1 dargestellt. Hierbei wurde besonderer Wert auf eine ausreichend lange Fahrtdauer und monotone Fahrbedingungen gelegt, da die

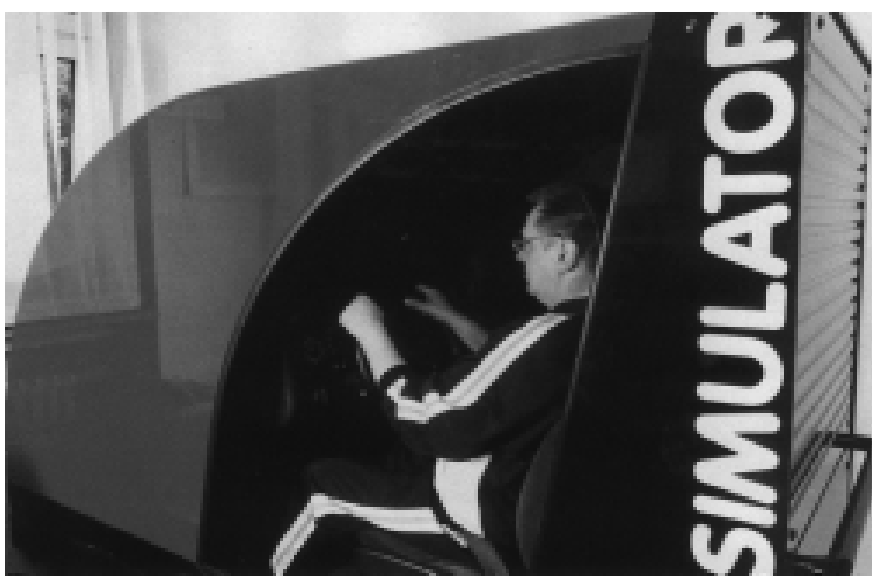

Abb. 1 Fahrsimulator C.A.R. 
Tab. 1 Fahrtbedingungen in der simulierten Fahrsituation

\begin{tabular}{ll}
\hline Fahrtdauer: & 60 Minuten \\
\hline Ort: & überwiegend Landstraße \\
\hline mittlere Geschwindigkeit: & $100 \mathrm{~km} / \mathrm{h}$ \\
\hline unterschiedliche Witterung: & Regen, Nebel, Schnee, Schönwetterfahrt \\
\hline unterschiedliche Tageszeiten: & Tag-, Dämmerungs-, Nachtfahrt \\
wenig Hindernisse: & Reh, Fußgänger, andere Fahrzeuge
\end{tabular}

Mehrzahl der Einschlafattacken unter diesen Umständen auftritt.

\section{Statistik}

Zur Analyse der erhobenen Daten dienten das Programm Sigma Stat Version 2.0 von Jandel Scientific sowie SPSS Version 9.0.1 von SPSS. Es erfolgte eine Analyse der klinischen Daten mittels parametrischer Testverfahren: Student-t-Test für gepaarte (verbundene) Stichproben mit Normalverteilung bzw. WilcoxonSigned-Rank-Test bei nicht vorhandener Normalverteilung zur Erkennung von Therapieeffekten. Zum Gruppenvergleich zwischen Normal- und Patientenkollektiv dienten der t-Test für unverbundene Stichproben bei Normalverteilung bzw. der MannWhitney-Rank-Summen-Test bei nicht normal verteilten Daten. Statistische Signifikanz wurde bei einer Irrtumswahrscheinlichkeit von $\mathrm{p}<0,05$ angenommen.

\section{Ergebnisse}

Tab. 2 zeigt den Vergleich der anamnestischen Angaben mittels Fragebogen nach Siegrist [30] bzw. der Epworth Sleepiness Scale (ESS) zwischen Gesunden und Patienten vor und unter CPAP. Die OSAS-typischen Symptome (Tagesschläfrigkeit, Einschlafneigung, eingeschränkte Leistungfähigkeit) überwogen bei den Patienten. Zusätzlich wiesen Patienten mit OSAS einen pathologischen ESS-Score auf, der sich signifikant von den Gesunden unterschied. Unter CPAP besserten sich die OSAS-typischen Beschwerden im vorliegenden Kollektiv zwar nicht im Rahmen der Akuttherapie, sondern erst im mittelfristigen Verlauf nach sechs Wochen. Der ESS-Score nahm bereits nach 2 Tagen CPAP signifikant ab und besserte sich zusätzlich im weiteren Verlauf.
Tab. 3 zeigt die polysomnographischen Ergebnisse des Normkollektivs und der Patienten mit OSAS vor, 2 und 42 Tage nach eingeleiteter CPAP Therapie. Der REM-Anteil war im vorliegenden Patientenkollektiv höher als bei den Kontrollen, ohne das Signifikanz erreicht wurde. Insgesamt ist der REM-Anteil in beiden Patientenkollektiven niedrig, was u.U. durch die ungewohnte Messsituation begründet ist. Der Tiefschlafanteil lag in beiden Kollektiven im Normbereich, wobei der Tiefschlafanteil bei Normprobanden signifikant höher war als bei OSAS-Patienten. Der Wachanteil war in beiden Gruppen gegenüber der Norm erhöht. Der Definition des OSAS entsprechend zeigten sich deutlich Unterschiede im Hinblick auf die ventilatorischen Parameter Apnoe- und Apnoe-Hypopnoe-Index sowie die minimale Sauerstoffsättigung und die Zeitdauer der Entsättigungen $<90 \%$ (t90), die mittlere Sauerstoffsättigung unterschied sich nicht zwischen beiden Kollektiven. Unter CPAP wurde REM- und Tiefschlafanteil nicht beeinflusst, der Wachanteil nahm tendenziell, jedoch nicht signifikant, zu. Bereits nach 2 Tagen CPAP gelang eine signifikante Besserung der ventilatorischen Parameter und der Sauerstoffsättigung, die sich nach 6 Wochen bestätigte. Hinsichtlich der Schlafarchitektur kam es zu keiner Zunahme des REM- und Tiefschlafanteils, der Wachanteil nahm tendenziell im Laufe der Therapie ab. Bezüglich der ventilatorischen Parameter und der Sauerstoffsättigung konnte bereits im Rahmen der Akuttherapie eine signifikante Besserung unter CPAP erzielt werden; diese Ergebnisse bestätigten sich im mittelfristigen Verlauf.

Tab. 4 zeigt die Ergebnisse der Fahrsimulatoruntersuchung bei Gesunden und bei OSAS-Patienten bei Diagnosestellung, 2 und 42 Tage nach eingeleiteter CPAP-Therapie. Es zeigten sich signifikante Unterschiede zwischen OSAS-Patienten und Kontrollen bzgl. der Gesamtzahl der verursachten Unfälle, wobei OSAS-Patienten häufiger Auffahrunfälle verursachten. Konzentrationsfehler mit potenzieller Unfallfolge traten bei OSAS-Patienten ebenfalls signifikant häufiger auf. Die Durchschnittsgeschwindigkeit und die gefahrene Distanz unterschieden sich nicht zwischen beiden Kollektiven. Auch zeigten sich bei verschiedenen Tageszeiten keine Unterschiede der Unfallhäufigkeit zwischen Patienten und Kontrollen. Unter CPAP kam es zu einer signifikanten Reduktion der Gesamtzahl der Unfälle, Auffahrunfälle, Konzentrationsfehler sowohl im Rahmen der Akuttherapie als auch im Verlauf nach 6 Wochen. Die Abb. 2 und 3 stellen den Vergleich der Gesamtzahl der Unfälle und Konzentrationsfehler bei Gesunden und OSAS-Patienten vor und unter Therapie grafisch

Tab. 2 Anamnestische Angaben bei Normprobanden und OSAS-Patienten vor und unter CPAP. $\left({ }^{*} \mathrm{p}<0,05,{ }^{* *} \mathrm{p}<0,01,{ }^{* * *} \mathrm{p}<0,001,{ }^{*},{ }^{* *}\right.$, *** bezieht sich auf den Vergleich der jeweiligen Spalte zu OSAS vor CPAP)

\begin{tabular}{|c|c|c|c|c|}
\hline & $\begin{array}{l}\text { OSAS vor CPAP } \\
(n=31)\end{array}$ & $\begin{array}{l}\text { OSAS + CPAP (Tag 2) } \\
(n=24)\end{array}$ & $\begin{array}{l}\text { OSAS + CPAP (Tag 42) } \\
(n=21)\end{array}$ & $\begin{array}{l}\text { Normprobanden } \\
(n=10)\end{array}$ \\
\hline \multicolumn{5}{|l|}{ Fragebogen nach Siegrist } \\
\hline Einschlafneigung & $2,7 \pm 1,2$ & $2,3 \pm 1,2$ & $1,5 \pm 0,7^{* *}$ & $1,5 \pm 0,7^{* *}$ \\
\hline eingeschränkte Leistungsfähigkeit & $3,3 \pm 1,3$ & $2,9 \pm 0,9^{* *}$ & $2,1 \pm 1,1^{* * *}$ & $1,7 \pm 0,8^{* *}$ \\
\hline Schnarchen & $3,9 \pm 1,2$ & $3,9 \pm 0,9$ & $1,8 \pm 1,1^{* * *}$ & $3,6 \pm 0,9$ \\
\hline Epworth Score & $10,1 \pm 4,2$ & $8,9 \pm 4,5^{*}$ & $6,1 \pm 3,3^{* * *}$ & $6,6 \pm 3,6^{*}$ \\
\hline
\end{tabular}


Tab. 3 Polysomnographische und pulsoximetrische Messergebnisse bei Normprobanden und OSAS-Patienten vor und unter CPAP. $\left({ }^{*} \mathrm{p}<0,05,{ }^{* *} \mathrm{p}<0,01,{ }^{* * *} \mathrm{p}<0,001,{ }^{*},{ }^{* *},{ }^{* * *}\right.$ bezieht sich auf den Vergleich der jeweiligen Spalte zu OSAS vor CPAP)

\begin{tabular}{|c|c|c|c|c|}
\hline & $\begin{array}{l}\text { OSAS vor CPAP } \\
(n=31)\end{array}$ & $\begin{array}{l}\text { OSAS + CPAP (Tag 2) } \\
(n=24)\end{array}$ & $\begin{array}{l}\text { OSAS + CPAP (Tag 42) } \\
(n=21)\end{array}$ & $\begin{array}{l}\text { Normprobanden } \\
(n=10)\end{array}$ \\
\hline \multicolumn{5}{|l|}{ Schlaf } \\
\hline Gesamtschlafzeit (min) & $345,9 \pm 64,6$ & $347,4 \pm 53,6$ & $347,7 \pm 57,7$ & $393,9 \pm 9,2$ \\
\hline REM (\%) & $16,1 \pm 8,5$ & $17,4 \pm 9,0$ & $17,8 \pm 6,2$ & $12,9 \pm 5,0$ \\
\hline Tiefschlaf (\%) & $9,9 \pm 8,7$ & $12,1 \pm 7,9$ & $10,9 \pm 8,3$ & $18,5 \pm 6,1^{* *}$ \\
\hline Wachanteil (\%) & $10,3 \pm 9,9$ & $8,3 \pm 9,9$ & $6.3 \pm 7,7$ & $8,4 \pm 5,6$ \\
\hline \multicolumn{5}{|l|}{ Atmung/Sauerstoffsättigung } \\
\hline Apnoe-Hypopnoe-Index (/h) & $24,8 \pm 21,5$ & $1,2 \pm 2,0^{* * *}$ & $0,7 \pm 0,9^{* * *}$ & $5,6 \pm 6,3^{* * *}$ \\
\hline $\mathrm{S}_{a} \mathrm{O}_{2 m}(\%)$ & $93,5 \pm 3,1$ & $95,3 \pm 0,8^{* * *}$ & $95,1 \pm 0,9^{* * *}$ & $94,3 \pm 2,6$ \\
\hline $\mathrm{S}_{a} \mathrm{O}_{2 \min }(\%)$ & $80,5 \pm 10,9$ & $86,3 \pm 17,9^{* * *}$ & $88,4 \pm 5,0^{* * *}$ & $90,0 \pm 2,3^{* * *}$ \\
\hline t90 (\%) & $6,9 \pm 12,5$ & $0,5 \pm 0,8^{* * *}$ & $1,1 \pm 1,9^{* *}$ & $0,2 \pm 0,2^{* * *}$ \\
\hline
\end{tabular}

Tab. 4 Fahrsimulatoruntersuchung bei Normprobanden und OSAS-Patienten vor und unter CPAP. $\left({ }^{*} \mathrm{p}<0,05,{ }^{* *} \mathrm{p}<0,01,{ }^{* * *} \mathrm{p}<0,001,{ }^{*},{ }^{* *}\right.$, *** bezieht sich auf den Vergleich der jeweiligen Spalte zu OSAS vor CPAP)

\begin{tabular}{|c|c|c|c|c|}
\hline & $\begin{array}{l}\text { OSAS vor CPAP } \\
(n=31)\end{array}$ & $\begin{array}{l}\text { OSAS + CPAP (Tag 2) } \\
(n=24)\end{array}$ & $\begin{array}{l}\text { OSAS + CPAP (Tag 42) } \\
(n=21)\end{array}$ & $\begin{array}{l}\text { Normprobanden } \\
(n=10)\end{array}$ \\
\hline Gesamtzahl Unfälle & $2,7 \pm 2,0$ & $1,5 \pm 1,4^{* *}$ & $0,9 \pm 1,3^{* *}$ & $1,3 \pm 1,5^{*}$ \\
\hline Auffahrunfälle & $1,9 \pm 1,7$ & $0,9 \pm 1,2^{*}$ & $0,8 \pm 1,0^{* *}$ & $0,8 \pm 0,9^{*}$ \\
\hline Konzentrationsfehler & $12,4 \pm 5,1$ & $6,5 \pm 3,9^{* *}$ & $4,9 \pm 3,3^{* *}$ & $7,1 \pm 3,2^{* *}$ \\
\hline \multicolumn{5}{|c|}{ Unfälle bei versch. Wetterlagen/Tageszeiten } \\
\hline Nebel & $0,2 \pm 0,5$ & $0,1 \pm 0,3$ & $0,1 \pm 0,3$ & $0,1 \pm 0,3$ \\
\hline Schnee & $0,2 \pm 0,5$ & $0,0 \pm 0,2$ & $0,2 \pm 0,4$ & $0,1 \pm 0,3$ \\
\hline Nacht & $0,6 \pm 0,8$ & $0,3 \pm 0,6$ & $0,1 \pm 0,2$ & $0,3 \pm 0,7$ \\
\hline gefahrene Distanz (km) & $51,1 \pm 8,6$ & $47,9 \pm 8,3$ & $52,6 \pm 7,6$ & $53,1 \pm 8,8$ \\
\hline Durchschnittsgeschwindigkeit (km/h) & $51,4 \pm 8,8$ & $48,7 \pm 8,2$ & $52,6 \pm 7,6$ & $53,1 \pm 8,8$ \\
\hline
\end{tabular}

dar. Unter CPAP gelingt eine Absenkung beider Faktoren unter den Messwert bei Gesunden.

\section{Diskussion}

Neben einer individuellen Gefährdung aufgrund einer erhöhten Prävalenz kardiovaskulärer Erkrankungen [31-37] stellt das OSAS auch eine kollektive Gefährdung dar. Diese resultiert aus den Auswirkungen des OSAS auf die subjektive Tagesbefindlichkeit, bei der Müdigkeit und Einschlafneigung mit konsekutiv erhöhter Unfallgefahr im Vordergrund stehen. Es konnte nachgewiesen werden, dass das Unfallrisiko bei OSAS im Vergleich zu Gesunden um das mindestens 2 - 3fache bis zum 7 fachen erhöht ist [5 - 13]. Da diese erhöhte Unfallneigung nicht nur die Teilnahme am Straßenverkehr, sondern vor allem auch den Arbeitsplatz betrifft, ist das OSAS auch arbeitsmedizinisch von großer Bedeutung. Die Erfassung und Quantifizierung der Unfallneigung und -häufigkeit bei OSAS ist insbesondere im Hinblick auf die Beurteilung sozialmedizinischer und gutachterlicher Fragestellungen wie z. B. der Fahrtüchtigkeit und der Arbeitsfähigkeit des Patienten erforderlich. Die Erfassung der Unfallhäufigkeit erfolgt an- hand von anamnestischen Angaben, Unfallstatistiken und Fahrsimulatoruntersuchungen. Anamnese und Unfallstatistiken bergen das Risiko der Unterschätzung der tatsächlichen Unfallhäufigkeit, da viele Patienten aus Angst vor beruflichen Sanktionen wie z.B. Arbeitslosigkeit ihre tatsächliche Unfallneigung verschweigen.

Fahrsimulatoren werden zunehmend zur Evaluierung der Unfallhäufigkeit bei OSAS vor und unter Therapie eingesetzt. Der Vorteil von Fahrsimulatoren besteht in dem fehlenden Risiko sowohl für den Untersucher als auch für den Untersuchten. Die in der Literatur beschriebenen Fahrsimulatoren testen jedoch zumeist nicht den Aufmerksamkeitsaspekt der Vigilanz, d.h. der Aufrechterhaltung der Aufmerksamkeit unter monotonen Umgebungsbedingungen, sondern die Aufmerksamkeitskomponente der geteilten Aufmerksamkeit (z.B. DADT) bzw. die Daueraufmerksamkeit (z. B. Steer clear) $[7,9,10]$.

Der in der vorliegenden Untersuchung angewandte Fahrsimulator C.A.R. ermöglicht eine realitätsnahe, ausreichend lange Fahrsimulation unter Monotoniebedingungen und erfasst somit den Aufmerksamkeitsaspekt der Vigilanz, der bei länger dauernden 


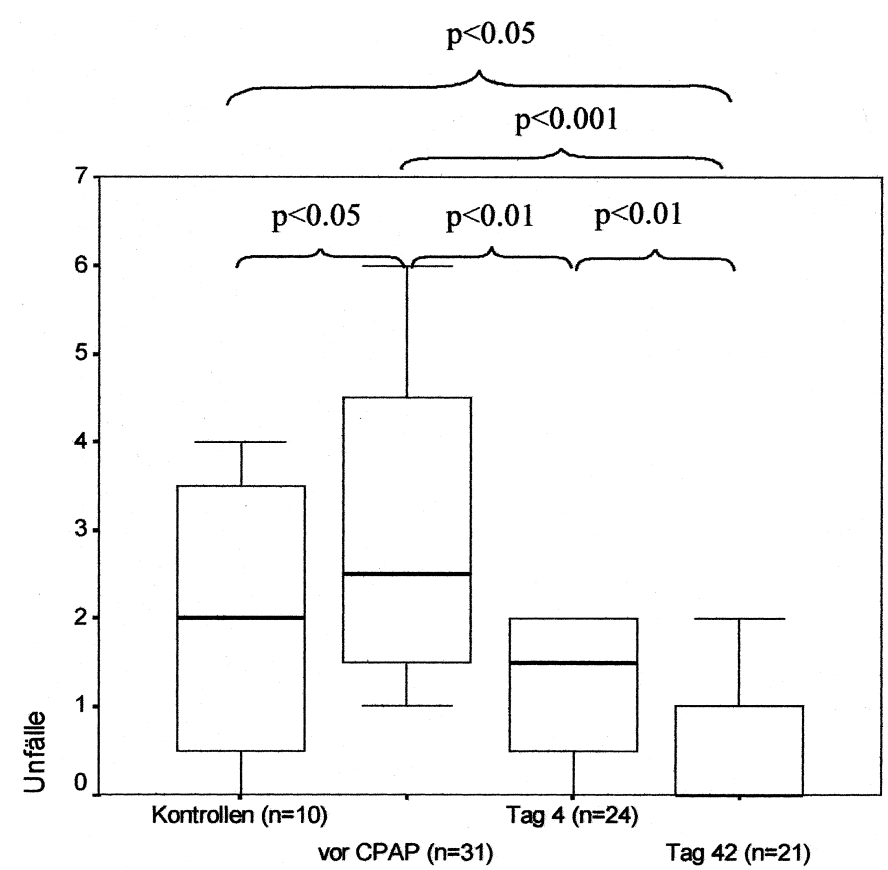

Abb. 2 Boxplot der Unfallhäufigkeit in der simulierten Fahrsituation bei Kontrollen und Patienten mit OSAS vor und unter CPAP.

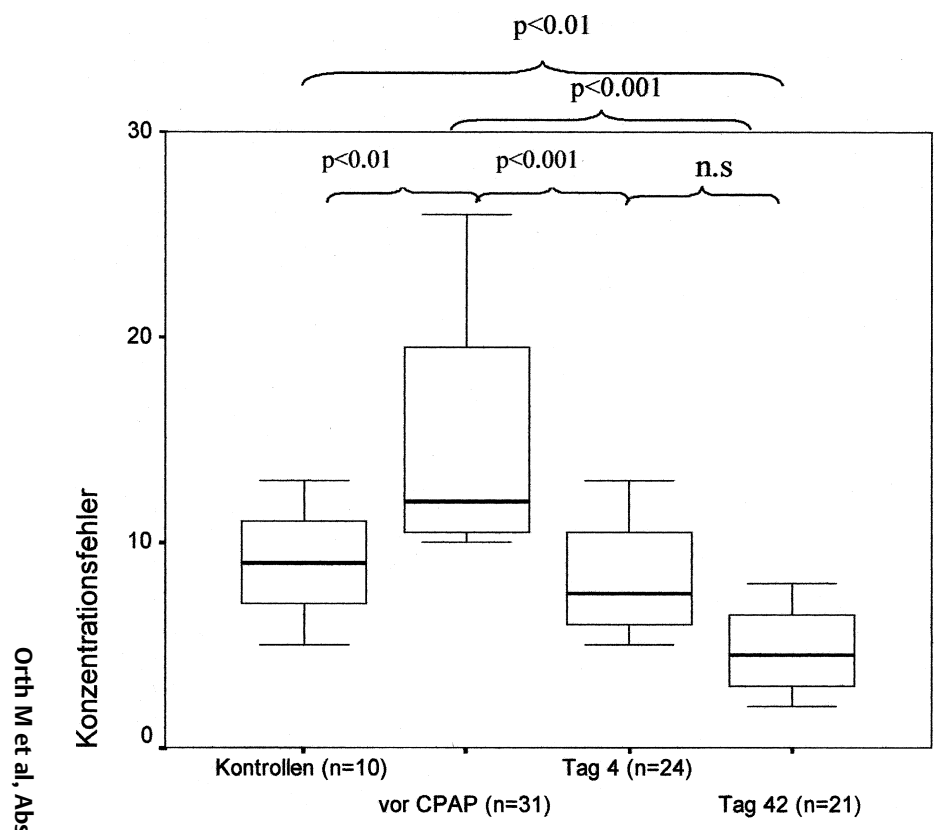

Abb. 3 Boxplot der Häufigkeit von Konzentrationsfehlern in der simulierten Fahrsituation bei Kontrollen und Patienten mit OSAS vor und unter CPAP.

Autofahrten und wenig Verkehrsdichte gefordert ist. Der Vergleich der Fahrsimulatorleistung von OSAS-Patienten und Gesunden zeigt in der vorliegenden Untersuchung signifikante Unterschiede im Hinblick auf die Gesamtzahl der Unfälle, wobei die Auffahrunfälle im Vordergrund stehen. Die Literaturangaben einer bei OSAS um das mindestens 2 - 3fach erhöhten Unfallhäufigkeit gegenüber Gesunden bestätigen sich anhand der Ergebnisse. Auch die Konzentrationsfehler, die zu Beinahe-Unfällen bzw. tatsächlichen Unfällen führen können, sind im vorliegenden Kollektiv von OSAS-Patienten gegenüber dem Normkollektiv erhöht. Die Unterschiede im Fahrverhalten zwischen Patienten und Normprobanden bessern sich unter Therapie und gleichen sich den Werten der Normalpersonen an. Bereits nach 2 Nächten CPAP kommt es zu einer signifikanten Absenkung der Gesamtzahl der Unfälle, der Auffahrunfälle und der Konzentrationsfehler. Nach 6 Wochen lässt sich nochmals eine weitere Besserung der Unfallhäufigkeit und der Konzentrationsfehler in der simulierten Fahrsituation feststellen. Letztendlich kann anhand der vorliegenden Ergebnisse nicht ausgeschlossen werden, dass der Therapieerfolg nach 2 Nächten CPAP-Therapie auf einem Lerneffekt der zwei Tage zuvor stattgehabten Untersuchung beruht, ein nach sechs Wochen anhaltender Lernerfolg als Ursache für die weitere Besserung der Fahrsimulatorleistung kann zwar ohne einen entsprechenden Vergleich mit der Kontrollgruppe nicht sicher ausgeschlossen werden, erscheint jedoch unwahrscheinlich. Die vorliegende Fahrsimulatoruntersuchung bestätigt somit die Ergebnisse anderer Fahrsimulatoruntersuchungen, die zum einen signifikante Unterschiede in der Fahrsimulatorleistung zwischen OSAS-Patienten und Gesunden sowie eine relevante Absenkung der Fehlerhäufigkeit unter adäquater Therapie bestätigen können $[7,21-28,30]$.

Der vorgestellte Fahrsimulator C.A.R. ist in der Lage, die intraindividuelle Unfallgefährdung vor bzw. unter Therapie abzuschätzen. Da möglichst realitätsnahe Fahrsimulatoren einen hohen finanziellen und personellen Aufwand bedeuten, ist die Integration von Fahrsimulatoruntersuchungen in das routinemäßige diagnostische Spektrum müdigkeits- und schläfrigkeitsbezogener Symptome schwer realisierbar. Empfehlenswert ist jedoch die Integration einer Fahrsimulatoruntersuchung in das diagnostische Management der Beurteilung der Fahrtüchtigkeit bei arbeitsmedizinischen und gutachterlichen Fragestellungen wie z.B. Fahrtüchtigkeit sowie zur Beurteilung der Fahrtüchtigkeit bei Patienten mit trotz optimaler Therapie fortbestehender Einschlafneigung bzw. erhöhter Unfallneigung.

\section{Literatur}

${ }^{1}$ Langwieder K, Sporner A, Hell W. Struktur der Unfälle mit Getöteten auf Autobahnen im Freistaat Bayern im Jahr 1991. München: HUKVerband, Büro für Kfz-Technik, 1994

${ }^{2}$ Leger D. The cost of sleep-related accidents: a report for the National Commission on Sleep Disorders Research. Sleep 1994; 17: 84- 93

3 American Thoracic Society. Sleep apnea, sleepiness, and driving risk. Am J Respir Crit Care Med 1994; 150: 1463 - 1473

${ }^{4}$ McNicholas WT. Sleep apnoea and driving risk. Eur Respir J 1999; 13 : $1225-1227$

${ }^{5}$ Young T, Blustein J, Finn L, Palta M. Sleep-disordered breathing and motor vehicle accidents in a population-based sample of employed adults. Sleep 1997; 20 (8): 608 -613

${ }^{6}$ Barbé F, Pericás J, Munoz A, Findley LJ, Anto JM, Agusti AGN, de Lluc Joan M. Automobile accidents in patients with sleep apnea syndrome. Am J Respir Crit Care Med 1998; 158: 18 - 22

${ }^{7}$ Findley LJ, Fabrizio MJ, Knight H, Norcross BB, Laforte AJ, Suratt PM. Driving simulator performance in patients with sleep apnea. Am Rev Respir Dis 1989a; 140: 529-530

8 Findley LJ, Smith C, Hooper J, Dineen M, Suratt PM. Treatment with nasal CPAP decreases automobile accidents in patients with sleep apnea. Am J Respir Crit Care Med 2000; 161: 857-859

${ }^{9}$ George C, Nickerson P, Hanley P, Millar T, Kryger M. Sleep apnea patients have more automobile accidents. Lancet 1987; 2 (8556): A447

10 George CFP, Smiley A. Sleep apnea and automobile crashes. Sleep 1999; 22: $790-795$

${ }^{11}$ Haraldsson PO, Carenfelt C, Diderichsen F, Nygren A, Tingvall C. Clinical symptoms of sleep apnea syndrome and automobile accidents. ORL 1990; 52: 57-62

12 Horne JA, Reyner LA. Sleep related vehicle accidents. BMJ 1995; 310: 565-567 
${ }^{13} \mathrm{Wu} \mathrm{H}$, Yan-Go F. Self-reported automobile accidents involving patients with obstructive sleep apnea. Neurology 1996; 46: 1254-1257

${ }^{14}$ Engleman HM, Hirst WSJ, Douglas NJ. Under reporting of sleepiness and driving impairment in patients with sleep apnoea/hypopnoea syndrome. J Sleep Res 1997; 6: 272-275

15 George CFP, Flaherty BA, Smiley A. Driving and sleep apnea, self-reported accidents. Sleep Res 1995; 24: A305

${ }^{16}$ Findley LJ, Suratt PM, Dinges DF. Time-on-task decrements in „steer clear" performance of patients with sleep apnea and narcolepsy. Sleep 1999; 22: 804-809

${ }^{17}$ Moscowitz H, Burns M. The effects of alcohol and valium, singly and in combination, upon driving-related skill performance. Proceedings of the $21^{\text {st }}$ Conference of the Association for Automobile Medicine. Vancouver: BC, 1977: 226-240

${ }^{18}$ Hartje W, Poeck K. Klinische Neuropsychologie. Stuttgart: Thieme, 1997: 59-79

${ }^{19}$ Posner MI, Rafael RD. Cognitive theories of attention and the rehabilitation of attentional deficits. In: Meier MJ, Benton AL, Diller L (eds.). Neuropsychological rehabilitation Edingbourgh: Churchill Livingston, 1987: $182-201$

${ }^{20}$ Büttner A, Randerath W, Rühle KH. Der Fahrsimulatortest „carsim“ zur Erfassung der Vigilanz. Einfluss der Fahrpraxis und weiterer Faktoren bei Gesunden und Patienten mit Schlafapnoe-Syndrom. Pneumologie 2000; $54: 338-344$

${ }^{21}$ Findley LJ, Fabrizio M, Thommi G, Suratt PM. Severity of sleep apnea and automobile crashes. N Engl J Med 1989b; 320: 868 - 869

${ }^{22}$ Findley LJ, Unverzagt M, Guchu R, Fabrizio M, Buckner J, Suratt P. Vigilance and automobile accidents in patients with sleep apnea or narcolepsy. Chest 1995; 108: 619-624

${ }^{23}$ Findley LJ, Weiss WJ, Jabour ER. Drivers with untreated sleep apnea: a cause of death and serious injury. Arch Int Med 1991; 151: 1451-1452

${ }^{24}$ George CFP, Boudreau AC, Smiley A. Effects of nasal CPAP on simulated driving performance in patients with obstructive sleep apnea. Thorax 1997; 52: 628-653

25 George CFP, Boudreau AC, Smiley A. Simulated driving performance in patients with obstructive sleep apnea. Am J Respir Crit Care Med 1996; 154: $175-181$
${ }^{26}$ Gerdesmeyer C, Randerath W, Rühle KH. Zeitliche Abhängigkeit der Fehlerzahl bei Messungen der Daueraufmerksamkeit mittels Fahrsimulator vor und nach nCPAP-Therapie bei Schlafapnoesyndrom. Somnologie 1997; 1 : $165-170$

${ }^{27}$ Haraldsson PO, Carenfelt C, Laurell H, Törnros J. Driving vigilance simulator test. Acta Otolaryngol 1990; 110: $136-140$

${ }^{28}$ Risser MR, Ware JC, Freeman FG. Driving simulation with EEG monitoring in normal and obstructive sleep apnea patients. Sleep 2000; 23: $393-398$

${ }^{29}$ Ruehle KH, Mayer G. Empfehlungen zur Begutachtung von SchlafWachstörungen und Tagesschläfrigkeit. Somnologie 1998; 2: 89-95

${ }^{30}$ Siegrist J, Peter JH, Himmelmann J, Geyer S. Erfahrungen mit einem Anamnesebogen zur Diagnostik der Schlafapnoe. Prax Klin Pneumol 1987; 41: 357-363

31 Bassetti C, Aldrich MS. Sleep apnea in acute cerebrovascular diseases. Sleep 1999; 22: $217-223$

${ }^{32}$ Hedner J, Ejnell $\mathrm{H}$, Caidahl K. Left ventricular hypertrophy independent of hypertension in patients with obstructive sleep apnoea. J Hypertens 1990; 8: 941 - 946

33 Millman RP, Redline S, Carlisle CC, Assaf AR, Levinson PD. Daytime hypertension in obstructive sleep apnea. Prevalence and contributing risk factors. Chest 1991; 99: 861 - 866

34 Podzus T, Bauer W, Mayer J, Penzel T, Peter JH, v Wichert P. Sleep apnea and pulmonary hypertension. Klin Wschr 1986; 64: 131-134

${ }^{35}$ Weitzenblum E, Krieger J, Apprill M, Valleé E, Ehrhart M, Ratomaharo J, Oswald M, Kurtz D. Daytime pulmonary hypertension in patients with obstructive sleep apnea syndrome. Am Rev Respir Dis 1988; 138: $345-349$

36 Worsnop CJ, Naughton MT, Barter CE, Morgan TD, Anderson AI, Pierce RJ. The prevalence of obstructive sleep apnea in hypertensives. Am J Respir Crit Cave Med 1998; 157: 111 - 115

37 Young T, Peppard P, Palta M, Hla KM, Finn L, Morgan B, Skatrud J. Population-based study of sleep-disordered breathing as a risk factor for hypertension. Arch Int Med 1997; 157: 1746-1752 